\title{
GPS in pollen tubes: Found!
}

\author{
Sha Li \& Yan Zhang* \\ State Key Laboratory of Crop Biology, College of Life Sciences, Shandong Agricultural University, Tai'an 271018, China
}

Received February 27; accepted March 1, 2016; published online March 4, 2016

Citation: Li, S., and Zhang, Y. (2016). GPS in pollen tubes: Found!. Sci China Life Sci 59, 438-439. doi: 10.1007/s11427-016-5032-7

Sexual reproduction of flowering plants depends on the delivery of two immotile sperm cells to the female gametophyte (FG), i.e. the embryo sac, through the growth of a pollen tube, a long cylindrical cellular extension from a pollen grain. The journey of pollen tubes toward the embryo sacs is led by female cues, which guide the path of the pollen tubes. Recognition between the male-female gametophytes in some cases precedes successful fertilization whereas in other cases serves as a reproduction isolation barrier. The century-old question thus followed: what signals are emitted by the female and what components in pollen tubes interpret them into directional growth?

A few years ago, a study using laser ablation on Torenia fournieri, a plant with protruding embryo sacs, demonstrated that LURE, a cysteine-rich polypeptide in a subgroup of defensin-like proteins, functions as the synergid-derived female attractant (Okuda et al., 2009). It answered the first half of the big question in sexual reproduction of flowering plants. The next obvious puzzle to solve is to identify the LURE receptors, whose role is comparable to that of Global Positioning System (GPS) such that they perceive LURE and interpret it into intracellular changes to guide growth direction.

The receptors of LURE have recently been identified by the research group led by Wei-Cai Yang, Institute of Genetics and Developmental Biology, Chinese Academy of Sciences. They designed a few criteria to select candidates for LURE receptors (Wang et al., 2016). First, the receptors are most probably receptor-like kinases (RLKs). RLKs resemble metazoan receptor tyrosine kinases in domain or-

*Corresponding author (email: yzhang@sdau.edu.cn) ganization, whose diverse extracellular domains (ECDs) are responsible for sensing distinct environmental cues (Shiu and Bleecker, 2001). Second, the RLK-coding genes should be expressed preferentially in pollen or pollen tubes. Third, genetic interference of the RLKs either by a loss-of-function or dominant-negative (DN) approach would compromise male transmission by reducing guidance efficiency of pollen tubes. Fourth, receptors for LURE would dimerize because signaling relay from RLKs often involves oligomerization. Based on these criteria, the authors were able to select two interacting pairs of RLKs, designated as MDIS1/2 (MALE DISCOVERER1/2) and MIK1/2 (MDIS1-INTERACTING RECEPTOR LIKE KINASE1/2), as candidates for the LURE receptors (Wang et al., 2016).

Wang et al. then performed extensive biochemical experiments to demonstrate that MDIS1/2 and MIK1/2 form the bona fide receptor complexes for LURE (Wang et al., 2016). They showed that LURE binds to the ECDs of MDIS1 and MIK1/MIK2 by pull-down, microscale thermophoresis, and co-immunoprecipitation (co-IP) assays. Using the same approaches, Wang et al. also demonstrated the direct interaction between the ECDs of MDIS1 and MIK1 or MIK2. Interaction of these co-receptors was enhanced by exogenously applied LURE, implying LURE-induced receptor oligomerization. Because signal relay from RLKs accompanies the phosphorylation of their intracellular domains (Shiu and Bleecker, 2001), the authors further analyzed whether that was the case for the LURE receptors. Indeed, they were able to identify both auto-phosphorylation and trans-phosphorylation by MIK1. They additionally pinpointed a few phosphorylation sites within the intracellular domains (ID) of MDIS1 and MIK1, although the functional relevance of these phosphorylation 
sites is yet to be characterized. In addition, LURE induces the phosphorylation of MDIS1 and MIK1, providing mechanistic support to their identities as the LURE receptors. Finally, Wang et al. also showed that expressing the Arabidopsis MDIS1 in Capsella rubella pollen tubes significantly enhanced their ability of directional growth toward Arabidopsis ovules, indicating a compromised reproduction barrier (Wang et al., 2016).

As for any other scientific breakthroughs, a great finding raises more questions than it answers. First, male transmission defects of the MDISI/2 and MIKI/2 mutants were not fully penetrant (Wang et al., 2016). Whether it is due to hierarchy redundancy or the presence of other receptor complexes is yet to be determined. In the scenario of the former, genomic editing technologies such as CRISPR/Cas9 will be useful. Second, the intracellular components through which MDIS1/2-MIK1/2 relay the directional cue are unknown. Replacing the ID of MDIS1 with an inactive counterpart of BRASSINOSTEROID INSENSITIVE1 rendered a DN version of MDIS1, implying that the MDIS1/2MIK1/2 receptor complex shares similar intracellular components with other RLKs. Because RLK-released autoinhibition of guanine nucleotide exchange factors (RopGEFs) (Zhang and McCormick, 2007) seems a widely-adopted intracellular signaling pathway in plant cells, the small GTPases, Rho of Plants (ROPs), are potential candidates for the directional growth of pollen tubes upon LURE perception. Third, RLKs are transmembrane proteins, which are synthesized and properly folded at the endoplasmic reticulum (ER), delivered via vesicles to the plasma membrane (PM), and possibly dynamically recycled within endomembrane compartments. Yang's group previously showed that a complex consisting of two ER proteins, POLLEN DEFECTIVE IN GUIDANCE1 and CALRETICULIN3, is critical for the FG-guided directional growth of pollen tubes
(Li et al., 2011). It waits to be seen whether the ER complex regulates the function of MDIS1/2-MIK1/2 complex. Furthermore, Wang et al. showed in this study that LURE induced the internalization of MDIS1 (Wang et al., 2016). Whether its internalization terminates the guidance signal or propagates the guidance signal from vesicles is unclear. Finally, micropylar targeting in Arabidopsis requires a priming step, i.e. the growth of pollen tubes through the style. Whether and how the MDIS1/2-MIK1/2 complex is regulated by the priming process will be of great interest. These and more questions, provoked by the study of Wang et al., certainly expand the horizon of future researches on plant sexual reproduction.

Compliance and ethics The author(s) declare that they have no conflict of interest.

Okuda, S., Tsutsui, H., Shiina, K., Sprunck, S., Takeuchi, H., Yui, R., Kasahara, R.D., Hamamura, Y., Mizukami, A., Susaki, D., Kawano, N., Sakakibara, T., Namiki, S., Itoh, K., Otsuka, K., Matsuzaki, M., Nozaki, H., Kuroiwa, T., Nakano, A., Kanaoka, M.M., Dresselhaus, T., Sasaki, N., and Higashiyama, T. (2009). Defensin-like polypeptide LUREs are pollen tube attractants secreted from synergid cells. Nature 458, 357-361.

Wang, T., Liang, L., Xue, Y., Jia, P.F., Chen, W., Zhang, M.X., Wang, Y.C., Li, H.J., and Yang, W.C. (2016). A receptor heteromer mediates the male perception of female attractants in plants. Nature DOI:10.1038/nature16975

Shiu, S.H., and Bleecker, A.B. (2001). Plant receptor-like kinase gene family: diversity, function, and signaling. Sci STKE re22.

Zhang, Y., and McCormick, S. A distinct mechanism regulating a pollen-specific guanine nucleotide exchange factor for the small GTPase Rop in Arabidopsis thaliana. Proc Natl Acad Sci USA, 2007, 104: 18830-18835.

Li, H.J., Xue, Y., Jia, D.J., Wang, T., Shi, D.Q., Liu, J., Cui F, Xie, Q., Ye, D., and Yang, W.C. POD1 regulates pollen tube guidance in response to micropylar female signaling and acts in early embryo patterning in Arabidopsis. Plant Cell, 2011, 23: 3288-3302.

Open Access This article is distributed under the terms of the Creative Commons Attribution License which permits any use, distribution, and reproduction in any medium, provided the original author(s) and source are credited. 